\title{
Die Reform der bundesstaatlichen Finanzverfassung: historische Voraussetzungen und aktueller Handlungsbedarf
}

von Joachim Wieland

Der deutsche Föderalismus gilt als reformbedürftig. Inzwischen wurde eine zweite Föderalismuskommission von Bundestag und Bundesrat eingesetzt. Sie hat den Auftrag, Vorschläge zur Modernisierung der Finanzbeziehungen von Bund und Ländern zu entwickeln. Der Beitrag erläutert, dass Deutschland aufgrund seiner historischen Entwicklung als unvollkommener Bundesstaat einzuordnen ist, dem eine Tendenz zur Zentralisierung innewohnt. Sodann werden die Neuerungen der Föderalismusreform I aus dem Jahr 2006 dargestellt. Weiterhin diskutiert der Beitrag die Ziele der Föderalismusreform II. Im Gespräch sind die Einführung einer neuen verfassungsrechtlichen Grenze für die Staatsverschuldung, Abhilfemöglichkeiten bei Haushaltsnotlagen der Länder sowie die Einführung einer Bundessteuerverwaltung.

German federalism is considered to be in need of reform. Therefore, Bundestag and Bundesrat installed a second commission on the reform of the federal state organisation. Its task is to develop recommendations on how to modernise the financial relations between the Federation and the Länder. This article explains that Germany is an imperfect federal state. For historical reasons, it has a tendency to centralisation. The contribution discusses the reforms introduced due to the first reform stage in 2006. It outlines, furthermore, the objectives of the ongoing second reform stage and discusses several key issues, such as a new constitutional threshold for public debt, remedies in case of excessive Länder deficits (Haushaltsnotlagen) as well as the introduction of a federal tax administration.

\section{I. (Zwischen-)Bilanz}

Die Föderalismusreform II ist Ausdruck eines in Deutschland seit einigen Jahren von Politik und Wissenschaft, aber auch von den Medien geäußerten Unbehagens an der Verfassung des Bundesstaates. Das überrascht zumindest auf den ersten Blick. Die bundesstaatliche Ordnung, die die Organisation des deutschen 
Staates prägt, gilt in Europa durchaus als Exportmodell. ${ }^{1}$ Blickt man nach Großbritannien, Spanien oder Belgien, kann man in Europa vielfach eine Tendenz feststellen, Machtbefugnisse vom Zentralstaat auf Regionen oder autonome Gebiete zu verlagern. ${ }^{2}$ Diese Entwicklung verläuft keineswegs ohne Probleme. Die Schotten möchten gerne den wirtschaftlichen Nutzen aus den Erdölvorkommen vor ihrer Küste für sich behalten, die Basken und Katalanen wollen die Kompetenzen der Zentralregierung zu Gunsten eigener Autonomiebestrebungen begrenzen, die im Baskenland bis zum Separatismus reichen. Flamen und Wallonen leben in so angespannten Verhältnissen, dass nicht erst seit der letzten Wahl in Belgien von einer Staatskrise gesprochen wird.

Demgegenüber ist das Verhältnis zwischen Bund und Ländern in Deutschland harmonisch und friedlich. Die Gliederung des Bundes in Länder und die grundsätzliche Mitwirkung der Länder bei der Gesetzgebung des Bundes gelten als so selbstverständlich, dass ihre Gewährleistung in der sogenannten Ewigkeitsgarantie des Art. 79 Abs. 3 GG, die selbst dem verfassungsändernden Gesetzgeber einen Zugriff verbietet, ${ }^{3}$ in der Staatspraxis fast als überflüssig gelten kann. Die bundesstaatliche Ordnung Deutschlands wird zumindest im Ausland als Beispiel für eine gelungene Synthese von Einhalt und Vielfalt angesehen. ${ }^{4}$

Warum besteht in Deutschland dennoch weithin Übereinstimmung, dass die bundesstaatliche Ordnung modernisierungsbedürftig ist? Ein Grund dürfte die Kritik am kooperativen Bundesstaat sein, der durch die Reform der Finanzverfassung in den Jahren 1968 und 1969 seinen Platz in der deutschen Verfassungsordnung gefunden hat. ${ }^{5}$ Die Verfechter eines Wettbewerbsföderalismus werfen dem kooperativen Bundesstaat vor, dass er die Vielfalt ersticke und damit den Bundesstaat eines wesentlichen Vorteils beraube. Erst wenn zwischen den Ländern Wettbewerb herrsche, könnten sich die Vorteile des Bundesstaates voll entfalten. Jedes Land könnte seinen Weg zur Verwirklichung des Gemeinwohls

1 Bauer, H., in: Dreier, H. (Hg.): Grundgesetz: Kommentar, Bd. 2, 2. Aufl., Tübingen, 2006, Art. 20 (Bundesstaat), Rn. 16; Kloepfer, M.: Bemerkungen zur Föderalismusreform, in: Die öffentliche Verwaltung, 57/13 (2004), 566-571, hier 571.

2 Hierzu die Berichte in: Piazolo, M./Weber, J. (Hg.): Föderalismus - Leitbild für die Europäische Union, München, 2004.

3 Vgl. Dreier, H., in: ders. (Hg.): Grundgesetz: Kommentar, Bd. 2, 2. Aufl., Tübingen, 2006, Art. 79 Abs. 3, Rn. 7; Jestaedt, M.: Bundesstaat als Verfassungsprinzip, in: Isensee, J./Kirchhof P. (Hg.): Handbuch des Staatsrechts, Bd. 2, 3. Aufl., Heidelberg, 2004, § 29, Rn. 48.

4 Vgl. Kloepfer, M., a.a.O., 567.

5 Zur Entwicklung der Finanzverfassung etwa Heun, W., in: Dreier, H. (Hg.): Grundgesetz: Kommentar, Bd. 3, Tübingen 2000, Vorbemerkung zu Art. 104-115, Rn. 6 ff. 
gehen, und die Bürger könnten zwischen den verschiedenen Modellen wählen. Es würde sich auf diesem Wege herausstellen, welche Problemlösungen sich bewährten und welche zum Scheitern verurteilt seien. Insgesamt werde im Wettbewerbsföderalismus die Effizienz des politischen Handelns gefördert, genauso wie in der Marktwirtschaft durch Wettbewerb der ökonomische Ertrag gesteigert werde. $^{6}$

Ein weiterer Kritikpunkt an der bestehenden bundesstaatlichen Ordnung wird darin gesehen, dass an die Stelle eines Gestaltungsföderalismus ein Beteiligungsföderalismus getreten sei. ${ }^{7}$ Die Länder hätten im Laufe der Jahre immer mehr eigene Kompetenzen an den Bund abgetreten und dadurch die Fähigkeit verloren, ihr Gemeinwesen nach den eigenen Vorstellungen zu gestalten. An die Stelle der ursprünglichen Autonomie sei das Recht zur Mitwirkung bei Entscheidungen des Bundes getreten. Markantestes Beispiel sei die Mitwirkung der Ministerpräsidenten der Länder an der politischen Willensbildung des Bundes im Bundesrat. Dadurch würden zwar die Befugnisse der Landesregierungen gestärkt, die Landtage jedoch entmachtet. An die Stelle der Autonomie der Länder trete eine Politikverflechtung zwischen Bund und Ländern. Sie führe dazu, dass es zu einer Vermischung der Verantwortung komme, weil dem Wähler als Souverän nicht mehr hinreichend deutlich werde, ob eine politische Entscheidung von Landespolitikern oder Bundespolitikern zu verantworten sei. Demokratie sei aber auf Entscheidungstransparenz angewiesen. Nur so könne demokratische Verantwortung eingefordert und realisiert werden. ${ }^{8}$

Hintergrund dieser Überzeugung vom Bedarf an Reformen der bundesstaatlichen Ordnung sind die Erfahrungen in der Regierungszeit der Kanzler Kohl und Schröder. In dieser Periode war es der Opposition im Bundesstaat jeweils relativ kurzfristig gelungen, die Mehrheit im Bundesrat zu erobern. Das ist eine Entwicklung, die für das deutsche politische System typisch ist. ${ }^{9}$ Bei Landtagswahlen tendieren die Wählerinnen und Wähler dazu, die Parteien zu bevorzugen, die

6 Vgl. Jun, U.: Reformoptionen der politischen Akteure im deutschen Föderalismus, in: Zeitschrift für Parlamentsfragen, 35/3 (2004), 559-580; Nettesheim, M.: Wettbewerbsföderalismus und Grundgesetz, in: Brenner, M./Huber, P.M./Möstl, M. (Hg.): Der Staat des Grundgesetzes - Kontinuität und Wandel, Festschrift für Peter Badura, Tübingen, 2004, 363-392.

7 Hierzu bereits Oeter, S.: Integration und Subsidiarität im deutschen Bundesstaatsrecht, Tübingen, 1998, $469 \mathrm{ff}$.

8 Vgl. Volkmann, U.: Bundesstaat in der Krise?, in: Die öffentliche Verwaltung, 51/15 (1998), 613-623, hier 618.

9 Hierzu auch Böckenförde, E.-W.: Sozialer Bundesstaat und parlamentarische Demokratie, in: ders.: Staat, Nation, Europa, 2. Aufl., Frankfurt, 2000, 183-207, hier 185 f. 
im Bund in der Opposition stehen. Das führte zu einem System von checks and balances, das im Sinne einer Machtkontrolle durchaus wünschenswert ist. Nachteilig ist allerdings die Versuchung für die Bundestagsopposition, die demokratisch legitimierte Regierungsmehrheit in ihrer politischen Arbeit permanent zu blockieren. ${ }^{10}$ Das führt zu einer faktisch dauerhaften großen Koalition im Vermittlungsausschuss. Nur das wird auf gesetzlicher Grundlage politisch verwirklicht, was sowohl der Regierung als auch der Opposition wünschenswert oder jedenfalls hinnehmbar erscheint. Regelmäßig erfolgt die Einigung auf den kleinsten gemeinsamen Nenner. Häufig werden auch sachfremde politische Entscheidungen in Paketen miteinander verkoppelt. Die Arbeit des Vermittlungsausschusses findet unter Ausschluss der Öffentlichkeit häufig in langen Nachtsitzungen statt und führt zu einer Entmachtung der Parlamente. ${ }^{11}$ Der Bundestag muss dem Entscheidungsvorschlag des Vermittlungsausschusses zwar noch zustimmen, kann ihn aber nicht abändern. Die Landesparlamente haben regelmäßig überhaupt keine Einwirkungsmöglichkeiten auf die Entscheidung über den Kompromissvorschlag des Vermittlungsausschusses im Bundesrat. Die Ministerpräsidenten müssen sich vor der Abstimmung im Bundesrat nicht rückversichern, ob die Landtagsmehrheiten den Kompromiss mittragen. Der Landtagsmehrheit wiederum fehlt die Zeit und nicht selten die Sachkunde, die Ministerpräsidenten von sich aus mit einer eigenen Position zu dem Vermittlungsausschussergebnis zu konfrontieren. Schließlich scheiterten viele Regierungsvorhaben im Bundesrat, weil Koalitionsregierungen in den Ländern regelmäßig vereinbart haben, sich im Bundesrat der Stimme zu enthalten, wenn zwischen ihnen in der Sache keine Einigung erzielt werden kann. Enthaltungen im Bundesrat wirken aber wie Neinstimmen, weil der Bundesrat seine Beschlüsse mit mindestens der Mehrheit seiner Stimmen fasst (Art. 52 Abs. 3 Satz 1 GG). ${ }^{12}$

Alle diese Umstände zusammen genommen begünstigen eine Politikverdrossenheit. Wählerinnen und Wähler haben in der Regierungszeit Kohl und Schröder oft den Eindruck gewonnen, dass ihre Stimmabgabe letztlich wenig bewirken

10 Ipsen, J.: Die Kompetenzverteilung zwischen Bund und Ländern nach der Föderalismusnovelle, in: Neue juristische Wochenschrift, 59/39 (2006), 2801-2806, 2802; Kesper, I.: Reform des Föderalismus in der Bundesrepublik Deutschland, in: Niedersächsische Verwaltungsblätter, 13/6 (2006), 145-158, 146.

11 Anschaulich Kühne, H.: Auslaufmodell Föderalismus?, München, 2004, 29 f.

12 Bauer, H., in: Dreier, H. (Hg.): Grundgesetz: Kommentar, Bd. 2, 2. Aufl., Tübingen, 2006, Art. 52, Rn. 19. 
konnte, weil alle relevanten Entscheidungen nur dann zustande gekommen sind, wenn sich die großen Parteien - unabhängig davon, ob sie im Bundestag in der Mehrheit oder in der Minderheit waren - auf ein Vorhaben geeinigt hatten. ${ }^{13}$ Es entstand zumindest der Eindruck, dass die Wahlbeteiligung unter diesem Zwang zur Einigung litt und dass notwendige Reformen nicht durchgeführt werden konnten.

Dem Bestreben um eine Modernisierung des Bundesstaates steht aber auch ein durchaus nicht geringes $\mathrm{Ma} ß$ an Skepsis gegenüber. ${ }^{14}$ Zum einen haben alle Beteiligten sich an die bestehende Ordnung gewöhnt, während jede Reform Unwägbarkeiten mit sich bringt und gegebenenfalls die politischen Akteure zur Anpassung an die dann neuen Gegebenheiten zwingt. Zum anderen ist aus naheliegenden Gründen das Interesse an einer Reform des Bundesstaates nicht überall gleich groß. Jede Beschneidung der Gesetzgebungskompetenzen des Bundes stößt fast zwangsläufig auf den Widerstand vieler Betroffener, der auch mehr oder weniger deutlich artikuliert wird. In den Bundesministerien führen weniger Gesetzgebungsbefugnisse des Bundes zum Wegfall von Referaten, Unterabteilungen oder sogar Abteilungen. Das ruft bei den betroffenen Ministern und bei ihren Beamten nicht nur Freude hervor. Lobbyisten pflegen lieber die Kontakte zu nur einem Ansprechpartner in Berlin statt zu 16 in den Landeshauptstädten. Wirtschaftsunternehmen sind schon aus Effizienzgründen an einem einheitlichen rechtlichen Rahmen für ihr Handeln interessiert. Ein gemeinsamer Markt drängt auf eine einheitliche Rechtsordnung, wie sich auch auf europäischer Ebene beobachten lässt. Im sozialen Bereich besteht ein kaum zu unterschätzendes Verlangen nach bundeseinheitlichen Regelungen. Die Sozialverbände befürchten von den Landesgesetzgebern tiefe Einschnitte in das soziale Netz und widersetzen sich deshalb vehement jeder Dezentralisierung der Gesetzgebungsbefugnisse.

Auch Bürgerinnen und Bürger identifizieren sich zwar mit ihren Ländern, in denen sie sich zu Hause fühlen. Um ihre Mobilität zu sichern, die schon der Arbeitsmarkt zwingend erfordert, sind sie jedoch an einem einheitlichen Rechtsrahmen für Schulen und Hochschulen interessiert und stehen der Forderung nach einer größeren Vielfalt der gesetzlichen Reglungen, die für ihr Leben von Bedeutung sind, ähnlich zurückhaltend gegenüber wie die Wirtschaftsunternehmen.

13 Vgl. auch Kühne, H., a. a. O., 20.

14 Siehe nur exemplarisch die Kontroverse um die Einführung einer Bundessteuerverwaltung, hierzu die Beiträge von Faltlhauser, K. und Hendricks, B., in: Die Welt vom 21.08. 2006, 10; Deutsche SteuerGewerkschaft, in: DSTG magazin, 5/2007, 5; Sarrazin, T., in: Der Tagesspiegel vom 16.09. 2007, 4. 
Die Gesetze der Länder zum Ladenschluss und zum Nichtraucherschutz sind nicht gerade Ausdruck eines Wettbewerbs der Ideen oder einer Begeisterung der Bevölkerung für unterschiedliche Lösungen in den einzelnen Ländern. ${ }^{15}$

Schließlich setzen sich zwar die finanzstärkeren Länder für mehr Autonomie in der Finanzwirtschaft ein. Die weitaus größere Zahl der finanzschwächeren Länder - nicht nur im Osten Deutschlands - will jedoch gerade wegen ihrer finanziellen Probleme möglichst an Gemeinschaftsaufgaben und Mischfinanzieren festhalten, von denen sie sich geldwerte Vorteile versprechen. Auch stehen finanzschwache und häufig hoch verschuldete Länder verfassungsrechtlichen Regelungen zur Begrenzung der Staatsverschuldung aus nachvollziehbaren Gründen skeptisch gegenüber, weil sie befürchten, politisch handlungsunfähig zu werden. ${ }^{16}$ Die Staatsfinanzen sollen selbstverständlich saniert werden. Dennoch ist die Bereitschaft der Bevölkerung, Sparmaßnahmen des Staates etwa in der Daseinsvorsorge hinzunehmen, relativ gering. Politiker erhöhen ihre Wahlchancen nicht dadurch, dass sie Sozialleistungen kürzen und weniger Geld für Straßen und Schienen, Kindergärten und Schulen, Krankenhäuser und Hochschulen ausgeben.

Das relativ abstrakte Bekenntnis zum Bundesstaat und zur Notwendigkeit seiner Modernisierung stößt also bei der konkreten Umsetzung auf erhebliche Widerstände und Schwierigkeiten. Ein wesentlicher Grund dafür dürfte in der geschichtlichen Entwicklung der bundesstaatlichen Ordnung in Deutschland liegen.

\section{Unvollkommener Bundesstaat}

Deutschland ist aufgrund seiner geschichtlichen Entwicklung ein unvollkommener Bundesstaat. ${ }^{17}$ Seit es in Deutschland eine föderalstaatliche Ordnung gibt, prägt eine Tendenz zur Zentralisierung die deutsche Geschichte. Nach dem Zerfall des Heiligen Römischen Reichs Deutscher Nation zu Beginn des 19. Jahrhunderts verfolgten Bürgerinnen und Bürger das Ziel, die staatliche Einheit der Nation herzustellen, wie sie etwa in Frankreich und Großbritannien seit

15 Siehe etwa die Kritik von Seils, C.: Staatlicher Flickenteppich, in: Zeit online vom 13.12. 2006, abrufbar unter http://www.zeit.de/online/2006/50/Foederalismus.

$16 \mathrm{Zu}$ den Finanzinteressen der Länder Härtel, I.: Föderalismusreform II, in: Juristenzeitung, 63/9 (2008), 437-446, hier 437.

17 Hierzu bereits Wieland, J.: Deutschlands Zukunft als Bundesstaat, in: Enders, C./Masing, J. (Hg.): Freiheit des Subjekts und Organisation von Herrschaft, Berlin, 2005, $82 \mathrm{ff}$. 
langem selbstverständlich war. ${ }^{18}$ Der 1815 auf dem Wiener Kongress gegründete Deutsche Bund bildete als Staatenbund ein Hindernis auf dem Weg zur deutschen Einheit. Er wurde von den Bürgerinnen und Bürgern als Relikt der alten Ordnung angesehen, die durch die Revolution von 1848/49 überwunden werden sollte. ${ }^{19}$ Nach deren Scheitern wurde 1871 durch die Gründung des Kaiserreichs die deutsche Einheit von oben hergestellt. Nach seiner Verfassung war das Kaiserreich ebenso wie der Norddeutsche Bund von 1867 ein Bundesstaat. Das Kaiserreich wurde laut Präambel seiner Verfassung als ewiger Bund der Fürsten und freien Handelsstädte gegründet. In der Staatspraxis war aber das Kaiserreich als Nationalstaat nicht nur ein Verein von Einzelstaaten, sondern „Staat einer Nation“. ${ }^{20}$ Die Nation nahm an der Ausübung der Staatsgewalt durch den Reichstag zunehmend aktiver teil. Gleichlaufend setzte sich der Selbstbestimmungsanspruch der Nation gegenüber dem traditionell legitimierten Herrschaftsanspruch der Fürsten durch. Das zeigte sich sehr bald nach der Reichsgründung im Prozess der Herstellung der Rechtseinheit. Schon die Paulskirchenverfassung hatte 1849 der Reichsgewalt aufgegeben, ,durch die Erlassung allgemeiner Gesetzbücher über bürgerliches Recht, Handels- und Wechselrecht, Strafrecht und gerichtliches Verfahren, die Rechtseinheit im deutschen Volk zu begründen“21. Der Norddeutsche Bund hatte 1869 die Gewerbeordnung beschlossen. 1871 war das materielle Strafrecht vereinheitlicht worden. 1879 folgten mit Gerichtsverfassungsgesetz, Rechtsanwaltsordnung, Zivilprozessordnung, Strafprozessordnung und Konkursordnung die sogenannten Reichsjustizgesetze, und das Reichsgericht nahm seine Arbeit auf. 1900 war die Herstellung der Rechtseinheit in Deutschland mit dem Inkrafttreten des Bürgerlichen Gesetzbuches und Handelsgesetzbuches im Wesentlichen abgeschlossen. ${ }^{22}$

Wirtschaft und Gesellschaft entwickelten sich im letzten Drittel des 19. Jahrhunderts auf nationaler Ebene. Die Industrialisierung machte in Deutschland ebenso wenig an den Grenzen der Einzelstaaten halt wie die Konjunkturzyklen, die Arbeiterbewegung, die Aktivitäten der Verbände und der politischen Parteien, die sich schnell auf nationaler Ebene organisierten. Die Herstellung des moder-

18 Vgl. etwa Wahl, R.: Die Entwicklung des deutschen Verfassungsstaates bis 1866, in: Isensee, J./Kirchhof, P. (Hg.): Handbuch des Staatsrechts, Bd. 1, 3. Aufl., München, 2003, § 2, Rn. 5 ff.

19 Vgl. Huber, E. R.: Das Kaiserreich als Epoche verfassungsstaatlicher Entwicklung, in: Isensee, J./Kirchhof, P. (Hg.): Handbuch des Staatsrechts, Bd. 1, 3. Aufl., München, 2003, § 4, Rn. 8.

20 Ebd., Rn. 9.

$21 \S 64$ der Frankfurter Reichsverfassung von 1849.

22 Stern, K.: Das Staatsrecht der Bundesrepublik Deutschland, Bd. 5, München, 2000, $417 \mathrm{ff}$. 
nen Staates der Wirtschaftsintervention vollzog sich zwar zunächst durch das Handeln der Einzelstaaten, mündete dann aber in die vom Reich zentral gelenkte Kriegswirtschaft des Ersten Weltkriegs, die einen in seiner Bedeutung nicht zu unterschätzenden Unitarisierungsschub mit sich brachte. ${ }^{23}$ Schließlich begründete die von Bismarck in den 80er Jahren des 19. Jahrhunderts initiierte Sozialversicherungsgesetzgebung die bis heute fortwirkende zentrale Ausrichtung des Sozialstaates gleich in seinen ersten Anfängen. ${ }^{24}$ Entscheidend gestärkt wurde der Zentralstaat im Übrigen durch den Primat des Reiches in der Außenpolitik und das persönliche Regiment von Kaiser Wilhelm II., nicht zuletzt durch seine Ausübung der Kommandogewalt, die faktisch die föderale Organisation des Heeres in Kontingente der Einzelstaaten überlagerte. ${ }^{25}$

Die Weimarer Reichsverfassung stärkte das Reich weiter gegenüber den Gliedstaaten. Die Staatsgewalt wurde nunmehr nicht durch einen Bund der Einzelstaaten, sondern durch das Deutsche Volk demokratisch legitimiert. Die Weimarer Reichsverfassung erwähnte das Bundesstaatsprinzip nicht einmal. Vielmehr mussten die Länder um die Anerkennung ihrer Staatlichkeit kämpfen. Das Reich verfügte in Gesetzgebung, Verwaltung, Rechtsprechung und in der Finanzordnung über sehr weitreichende Befugnisse, die besonders augenfällig in seiner eigenen Steuerhoheit verdeutlicht wurden. Die Vormachtstellung des Reiches gegenüber den Ländern war damit nicht nur organisatorisch, sondern finanzwirtschaftlich abgesichert. 1930 sprach die Staatsordnung deshalb von ,einer singulären bundesstaatlichen Rechtsordnung des labilen Föderalismus“ ${ }^{26}$ "Die Labilität erleichterte den Nationalsozialisten zunächst die Gleichschaltung und dann die faktische Auflösung der Länder. ${ }^{27}$

Nach dem Zweiten Weltkrieg errichteten die westlichen Alliierten die deutschen Länder wieder und sorgten für eine bundesstaatliche Ordnung. Die Länder schlossen sich nicht aus eigenem Antrieb zur Bundesrepublik Deutschland zusammen, vielmehr wurde deren Entstehung von den Militärgouverneuren zentral

23 Vgl. Huber, E. R.: Deutsche Verfassungsgeschichte seit 1789, Bd. 5, Stuttgart u. a., 1978, 73 ff.

24 Vgl. Zacher, H.F.: Das soziale Staatsziel, in: Isensee, J./Kirchhof, P. (Hg.): Handbuch des Staatsrechts, Bd. 2, 3. Aufl., München, 2004, § 28, Rn. 98.

25 Vgl. Huber, E. R.: Deutsche Verfassungsgeschichte seit 1789, Bd. 4, Stuttgart u. a., 1969, $330 \mathrm{f}$.

26 Thoma, R.: Das Reich als Bundesstaat, in: Anschütz, G./Thoma, R. (Hg.): Handbuch des Deutschen Staatsrechts, Bd. 1, Tübingen, 1930, 169-186, 184.

27 Stern, K., a. a. O., $784 \mathrm{ff}$. 
gesteuert. ${ }^{28}$ Soweit den Ländern Befugnisse zukamen, war die Stellung ihrer Exekutive beherrschend. Die Ministerpräsidenten waren auf deutscher Seite die Entscheidungsträger. Sie blieben es bis zur Gründung der Bundesrepublik und dem Inkrafttreten des Grundgesetzes. Das zeigte schon die Frankfurter Konferenz vom 1. Juli $1948 .{ }^{29} \mathrm{Zu}$ dieser Konferenz, mit der die Vorgeschichte des Grundgesetzes beginnt, wurden die Ministerpräsidenten von den drei westlichen Militärgouverneuren einbestellt. Die Ministerpräsidenten und nicht Abgeordnete aus den Landtagen verhandelten mit den Alliierten. Die Ministerpräsidenten entschieden in Absprache mit den Militärgouverneuren über die Modalitäten der Arbeit des Parlamentarischen Rates. Sie beriefen den Konvent von Herrenchiemsee ein. Während der Beratungen des Parlamentarischen Rates setzten sich vor allem die Militärgouverneure für eine Stärkung der Länderkompetenzen und gegen eine starke Bundesgewalt ein. Von deutscher Seite unterstützte nur das Land Bayern diese Bemühungen. Die Mehrheit im Parlamentarischen Rat wollte den Zentralstaat stärken. ${ }^{30}$ Mit diesen Bemühungen stieß sie auf den Widerstand der Alliierten. Das kam mehr als deutlich in einem Memorandum vom 2. März 1949 zum Ausdruck, mit dem die Militärgouverneure in großer Schärfe und kategorisch eine weitgehende Beschränkung der Kompetenzen des Bundes in der Gesetzgebung und in der Finanzverteilung forderten. Die Militärgouverneure setzten gegen den Willen der Mehrheit des Parlamentarischen Rats durch, dass die Bundesrepublik keine einheitliche Steuerverwaltung auf zentralstaatlicher Ebene erhielt, sondern dass dieses Machtinstrument zu einem wesentlichen Teil bei den Ländern verblieb. ${ }^{31}$ Nachdem deutlich wurde, dass an eine rasche Wiedervereinigung nicht zu denken war, führte der Kalte Krieg im Osten wie im Westen Deutschlands zu einer Stärkung der Zentralstaaten. Wirtschaftliche, soziale, partei- und verbandspolitische, bald auch außenpolitische und militärische Umstände legten in Westdeutschland ein Handeln des Bundes zumindest nahe. $^{32}$

Da die Ministerpräsidenten und nicht die Landtage auf deutscher Seite bei der Beratung des Grundgesetzes die bestimmende Rolle ausübten, überrascht die

28 Stolleis, M.: Besatzungsherrschaft und Wiederaufbau deutscher Staatlichkeit 1945-1949, 113 f., in: Isensee, J./Kirchhof, P. (Hg.): Handbuch des Staatsrechts, Bd. 1, 3. Aufl., München, 2003, § 7, Rn. $50 \mathrm{ff}$.

29 Hierzu Stern, K., a. a. O., $1214 \mathrm{ff}$.

30 Hierzu wiederum ebd., $1289 \mathrm{ff}$.

31 Vgl. dazu Oeter, S.: Die Finanzverwaltung im System der bundesstaatlichen Kompetenzverteilung, in: Thüringer Verwaltungsblätter, 6/1 (1997), 1-5.

32 Wieland, J., a. a. O., 87. 
Ausgestaltung ihrer Machtposition im Bundesrat nicht. Demgegenüber hatten die Landtage 1948/49 wenig Einfluss auf die bundesstaatliche Ordnung und konnten sich deshalb auch keine Machtbefugnisse sichern. Die Beschränkung der autonomen Gestaltungsmöglichkeiten der Landtage und die starke Stellung der Landesexekutiven waren damit strukturell in der deutschen Verfassungsordnung angelegt und zeichneten die weitere tatsächliche Entwicklung vor. ${ }^{33}$ Der ,unitarische Bundesstaat ${ }^{\star 34}$ ist nicht zufällig entstanden, sondern im Bundesstaatsprinzip deutscher Prägung angelegt. Diese verfassungsgeschichtliche Vorprägung der Bundesstaatlichkeit Deutschlands wird sich immer stärker erweisen als ökonomische Modelle eines idealen Bundesstaates oder als der Versuch, die aus einer anderen Geschichte entstandenen Bundesstaatsmodelle der Vereinigten Staaten von Amerika oder der Schweiz auf Deutschland zu übertragen. Das hat sich besonders deutlich bei der Umsetzung der Föderalismusreform I gezeigt.

\section{Föderalismusreform I}

Als Ergebnis der Föderalismusreform I sind die Befugnisse des Bundesgesetzgebers jedenfalls nicht sehr stark begrenzt worden. Man kann auch die Auffassung vertreten, dass sie bei einer Saldierung aller Änderungen des Grundgesetzes eher eine Stärkung erfahren haben. ${ }^{35}$ Dafür spricht vor allem die neue Fassung des Art. 72 Abs. 2 GG. Während der Bund früher im gesamten Bereich der konkurrierenden Gesetzgebung sein Gesetzgebungsrecht nur dann ausüben durfte, wenn und soweit die Herstellung gleichwertiger Lebensverhältnisse im Bundesgebiet oder die Wahrung der Rechts- oder Wirtschaftseinheit im gesamtstaatlichen Interesse eine bundesgesetzliche Regelung erforderlich machte, ist dieses Erforderlichkeitsgebot heute auf einen relativ kleinen Teil der in Art. 74 GG als Gegenstand der konkurrierenden Gesetzgebung aufgezählten Gesetzgebungskompetenzen beschränkt. ${ }^{36} \mathrm{Da}$ das Bundesverfassungsgericht in den vergangenen Jahren begonnen hatte, das Erforderlichkeitskriterium eher strikt zu verstehen

33 Berlit, U.: Verfassungsrechtliche Perspektiven des Föderalismus, in: Arnim, H.H.v. u.a. (Hg.): Föderalismus - Hält er noch, was er verspricht?, Berlin, 2000, 63-100, $68 \mathrm{ff}$.

34 Vgl. Hesse, K.: Der unitarische Bundesstaat, Karlsruhe, 1962; sowie Böckenförde, E.-W.: Sozialer Bundesstaat und parlamentarische Demokratie, in: ders.: Staat, Nation, Europa, 2. Aufl., Frankfurt, 2000, 183-207, hier $184 \mathrm{ff}$.

35 So etwa bzgl. des Bildungswesens Huber, P. M.: „Vom Kindergarten zur Habilitation“? - Der Bund als Gewinner der Föderalismusreform im Bildungswesen, in: Recht der Jugend und des Bildungswesens, 55/1 (2007), 4-7, hier 7.

36 Stettner, R., in: Dreier, H. (Hg.): Grundgesetz: Kommentar, Supplementum zu Band 2, Tübingen, 2007, Art. 72, Rn. 5 f. 
und dadurch die Gesetzgebungsbefugnisse des Bundes zu begrenzen, ${ }^{37}$ ist der Vorteil dieser Grundgesetzänderung für den Bund kaum zu unterschätzen. Demgegenüber sind die Begrenzungen der einzelnen Kompetenztitel im Bereich der konkurrierenden Gesetzgebung von nachrangiger Bedeutung. So hat der Bund im Bereich des Rechts der Wirtschaft nur die Kompetenz verloren, das Recht des Ladenschlusses, der Gaststätten, der Spielhallen, der Schaustellung von Personen, der Messen, der Ausstellungen und der Märkte zu regeln. Dabei handelt es sich um für das Wirtschaftsrecht eher periphere Gesetzgebungsbefugnisse. Die frühere Rahmengesetzgebung wurde in die konkurrierende Gesetzgebung überführt, so dass auch insoweit die Gesetzgebungsrechte des Bundes zunächst gestärkt wurden. ${ }^{38}$ Als Gegenleistung haben die Länder das Recht zur Abweichung von Bundesgesetzen in den Gebieten der früheren konkurrierenden Gesetzgebungszuständigkeit erhalten (Art. 72 Abs. 3 GG). Dieser Kompetenzgewinn hat durchaus seine Bedeutung. Allerdings wird erst die Staatspraxis zeigen, ob die Länder in der Lage sind, von ihren neuen Befugnissen auch kraftvoll Gebrauch zu machen.

Während der Bundesrat früher etwa zwei Dritteln der Bundesgesetze zustimmen musste, weil sie Reglungen über das Verwaltungsverfahren oder die Behördeneinrichtung enthielten, wurde dieses Zustimmungsrecht in der neuen Fassung des Art. 84 Abs. 1 GG begrenzt. ${ }^{39}$ Das Zustimmungsrecht des Bundesrates ist aber im Gegenzug dadurch ausgeweitet worden, dass Bundesgesetze, die Pflichten der Länder zur Erbringung von Geldleistungen, geldwerten Sachleistungen oder vergleichbaren Dienstleistungen gegenüber Dritten begründen und von den Ländern als eigene Angelegenheiten oder im Auftrag des Bundes ausgeführt werden, nunmehr gemäß Art. 104a Abs. 4 GG der Zustimmung des Bundesrates bedürfen, wenn daraus entstehende Ausgaben von den Ländern zu tragen sind. Welche Konsequenzen das in der Staatspraxis für die Reichweite des Zustimmungsrechts der Länder haben wird, wird sich verlässlich erst ermitteln lassen, wenn im Bund keine große Koalition mehr regiert, sondern einer die Bundesregierung tragenden

37 BVerfGE 106, 62 ff., hier 135 ff.; 110, 141 ff., hier 174 ff.; 111, 10 ff., hier 28 f.; 111, 226 ff., hier 254 f.

38 So auch die Einschätzung von Degenhart, C.: Die Neuordnung der Gesetzgebungskompetenzen durch die Föderalismusreform, in: Neue Zeitschrift für Verwaltungsrecht, 25/11 (2006), 1209-1216, hier 1212.

39 Vgl. Deutscher Bundestag, 16. Wahlperiode: Entwurf eines Gesetzes zur Änderung des Grundgesetzes, BT-Drs. 16/813 vom 07.03. 2006, $14 \mathrm{f}$. 
kleinen Koalition im Bundestag eine Bundesratsmehrheit gegenüber steht, die politisch von der Opposition im Bundestag gebildet wird. ${ }^{40}$

Auch die Mischfinanzierungstatbestände hat die Föderalismusreform I kaum verringert. ${ }^{41}$ Gestrichen wurde nur die Mitfinanzierung des Hochschulbaus durch den Bund. Zum Ausgleich wurde aber das Zusammenwirken von Bund und Ländern bei der Förderung von Einrichtungen und Vorhaben der wissenschaftlichen Forschung außerhalb von Hochschulen, bei Vorhaben der Wissenschaft und Forschung an Hochschulen und bei Forschungsbauten an Hochschulen einschließlich Großgeräten in Art. 91b Abs. 1 GG neu definiert. ${ }^{42}$

Wenn man nur die Föderalismusreform I betrachtet, besteht also eine erhebliche Diskrepanz zwischen der theoretischen Forderung nach einer Modernisierung des Bundesstaates und ihrer im Umfang relativ überschaubaren praktischen Durchsetzung. Der Grund für diese Diskrepanz liegt in der Eigenart der Bundesrepublik Deutschland als unvollkommener Bundesstaat, wie er sich in Deutschland in den letzten 200 Jahren entwickelt hat. Der Wunsch nach gleichwertigen Lebensverhältnissen war schon in der alten Bundesrepublik stark und ist durch die Wiedervereinigung weiter gestärkt worden. Die Ostdeutschen wollten vorrangig nicht bundesstaatliche Vielfalt, sondern gleichwertige Lebensverhältnisse wie im Westen Deutschlands. ${ }^{43}$ Eine verspätete und lange geteilte Nation wie Deutschland sieht die bundesstaatliche Vielfalt verständlicherweise eher skeptisch und weiß die Vorteile der Einheit stärker zu schätzen als andere Bundesstaaten mit einer weniger gebrochenen Geschichte. Hinzu kommt die harmonisierende Wirkung der Rechtsetzung der Europäischen Union. Sie lässt immer weniger Raum für Vielfalt auf Länderebene, sondern legt eine bundeseinheitliche Umsetzung der europarechtlichen Vorgaben häufig zumindest nahe. ${ }^{44}$

40 Selmer, P.: Die Föderalismusreform - Eine Modernisierung der bundesstaatlichen Ordnung, in: Juristische Schulung, 46/12 (2006), 1052-1060, hier $1057 \mathrm{f}$.

41 Vgl. Kesper, I., a. a. O., $151 \mathrm{f}$.

42 Hierzu Wollenschläger, F.: Die Föderalismusreform: Genese, Grundlinien und Auswirkungen auf die Bereiche Bildung und Wissenschaft, in: Recht der Jugend und des Bildungswesens, 55/1 (2007), 8-20, hier 18 .

43 Vgl. hierzu mit Hinweisen auf den Einigungsvertrag Badura, P.: Die innerdeutschen Verträge, insbesondere der Einigungsvertrag, in: Isensee, J./Kirchhof, P. (Hg.): Handbuch des Staatsrechts, Bd. 8, München, 1995, § 189, Rn. 30.

44 Vgl. Oeter, S.: Integration, a. a. O., $486 \mathrm{ff}$. 


\section{Perspektiven}

Dass Deutschland ein unvollkommener Bundesstaat ist, in dem bundesstaatliche Vielfalt zumindest in einem Spannungsverhältnis zum Ziel der Herstellung gleichwertiger Lebensverhältnisse steht, zeigen auch die Beratungen in der Kommission zur Modernisierung der Bund-Länder-Finanzbeziehungen. Ihre Empfehlungen sollen dazu beitragen, die Eigenverantwortung von Bund, Ländern und Kommunen zu stärken und für eine aufgabengerechte Finanzausstattung der Gebietskörperschaften zu sorgen. ${ }^{45}$ Im Mittelpunkt ihrer Arbeit steht die Suche nach einer neuen verfassungsrechtlichen Regel zur Begrenzung der Staatsverschuldung (1.), die Vorbeugung und Bewältigung von Haushaltsnotlagen (2.) und die Forderung des Bundes nach der Einführung einer Bundessteuerverwaltung (3.). Darüber hinaus beschäftigt sich die Kommission mit der Kritik der Staatsaufgaben und dem Setzen von Standards, mit der Entbürokratisierung und mit der Effizienzsteigerung der öffentlichen Verwaltung, die durch die Entflechtung von Aufgaben, eine Stärkung der den Aufgaben entsprechenden Finanzausstattung sowie der Eigenverantwortung der Gebietskörperschaften erreicht werden sollen. Erörtert werden auch die Möglichkeiten einer verstärkten Zusammenarbeit und eines erleichterten freiwilligen Zusammenschlusses von Ländern, die Bündelung fachpolitischer Leistungen und deren Auswirkungen auf die Bund-Länder-Finanzbeziehungen. Nach dem bisherigen Verlauf der Beratungen ist davon auszugehen, dass eine Einigung sich in vielen Bereichen als sehr schwierig erweisen wird. ${ }^{46}$ Vorschläge der Kommission sind am ehesten in den Bereichen Staatsverschuldung, Haushaltsnotlagen und möglicherweise Steuerverwaltung zu erwarten. Diese drei Themen werden im Folgenden näher in den Blick genommen.

\section{Verfassungsrechtliche Verschuldungsregel}

Das Grundgesetz begrenzt derzeit die Staatsverschuldung in Art. 115 Abs. 1 Satz 2 GG. Danach dürfen die Einnahmen aus Krediten die Summe der im Haushaltsplan veranschlagten Ausgaben für Investitionen nicht überschreiten. Ausnahmen sind nach der Verfassung nur zulässig zur Abwehr einer Störung des

45 Vgl. den Einsetzungsbeschluss Deutscher Bundestag, 16. Wahlperiode: Einsetzung einer gemeinsamen Kommission zur Modernisierung der Bund-Länder-Finanzbeziehungen, BT-Drs. 16/3885 vom 14.12. 2006.

46 Skeptisch bereits Selmer, P.: Zur Reform der bundesstaatlichen Finanzverfassung, Neue Zeitschrift für Verwaltungsrecht, 26/8 (2007), 872-880, hier 880. 
gesamtwirtschaftlichen Gleichgewichts. Diese Verschuldungsregel hat sich in den vergangenen 40 Jahren als wenig wirksam erwiesen. Der Schuldenstand der Gebietskörperschaften in Deutschland ist im Laufe der Zeit auf mehr als 1.500 Mrd. $€$ angestiegen. In der Folge geben Bund, Länder und Kommunen mehr als 65 Mrd. $€$ pro Jahr für Zinszahlungen aus. Das sind 12,5\% der Steuereinnahmen. ${ }^{47}$ Jeder achte Euro, den der Staat einnimmt, kann also nicht im Interesse des Gemeinwohls verwandt werden, sondern muss für Zinsen auf die hohe Staatsschuld aufgewandt werden.

In dieser Situation hatte der Bundesminister der Finanzen am 25. Februar der Föderalismuskommission II einen Vorschlag für eine neue verfassungsrechtliche Schuldenregel gemacht, die an den close-to-balance-Grundsatz des Europäischen Stabilitäts- und Wachstumspaktes anknüpft und eine maximale jährliche strukturelle Neuverschuldung von $0,5 \%$ des Bruttoinlandsprodukts erlaubt. ${ }^{48}$ Der Bund soll über einen Neuverschuldungsspielraum von $0,35 \%$ des Bruttoinlandsprodukts, die Ländergesamtgesamtheit über $0,15 \%$ des Bruttoinlandsprodukts verfügen. Außerdem soll die vorgeschlagene Regel eine symmetrische Berücksichtigung der konjunkturellen Situation ermöglichen. Sie soll also in Zeiten eines konjunkturellen Abschwungs eine zusätzliche Verschuldung zulassen und in Phasen des Aufschwungs für die Erzielung von Überschüssen sorgen bzw. zumindest die Kreditfinanzierungsspielräume verringern. ${ }^{49}$ Schließlich soll noch eine strenge Ausnahmeklausel geschaffen werden, die für die Handlungsfähigkeit des Staates, etwa bei Naturkatastrophen, Sorge trägt. Die Inanspruchnahme dieser Klausel soll nur dann erlaubt sein, wenn ein über die einfache Mehrheit hinausgehendes Quorum im Bundestag das billigt. Nach dem Vorschlag des Bundesfinanzministers soll ein Kontroll- und Sanktionsmechanismus gewährleisten, dass nicht von den genannten Regeln abgewichen wird..$^{50}$ Da das Bruttoinlandsprodukt gegenwärtig ca. $2.400 \mathrm{Mrd}$ € beträgt, würde der Vorschlag des Bundesfinanzministers eine strukturelle Neuverschuldung von insgesamt 12 Mrd. $€$ im Jahr erlauben, von denen 8,5 Mrd. $€$ auf den Bund entfielen. Demge-

47 Härtel, I., a. a. O., 437.

48 Sog. Steinbrück-Papier, Bundesministerium der Finanzen: Notwendigkeit und Inhalt einer neuen Schuldenregelung im Grundgesetz, K-Drs. 096 vom 25.02. 2008.

49 Ebd., $4 \mathrm{f}$.

50 Ebd., $6 \mathrm{f}$. 
genüber hat Art. 115 Abs. 1 Satz 2 GG in der Vergangenheit Kreditaufnahmen von 20 bis 30 Mrd. $€$ jährlich jedenfalls nicht verhindert. ${ }^{51}$

Die Steuerungswirkung der Finanzverfassung hat sich also in der Vergangenheit bei der Begrenzung der Staatsverschuldung als gering erwiesen. Während die Aufgabe der Finanzverfassung des Bundesstaates im Kern darin besteht, die Kompetenzen für die Steuergesetzgebung, die Steuerverwaltung und die Verteilung des Steuerertrags Bund und Ländern zuzuordnen und so für eine aufgabengerechte Finanzausstattung aller Gebietskörperschaften zu sorgen, gibt eine Verschuldungsregel ein inhaltliches Politikziel vor. ${ }^{52}$ Der Anreiz für die Politik, sich über die Verschuldungsregel der Verfassung mit mehr oder minder überzeugenden Erklärungen hinwegzusetzen, ist jedoch beachtlich. Wählerinnen und Wähler belohnen bei ihrer Wahlentscheidung regelmäßig nicht sie selbst treffende konkrete Sparentscheidungen, sondern belohnen Ausgaben im Bereich der gesamten Daseinsvorsorge, aus denen sie Nutzen ziehen. Das gilt für Sozialleistungen im weitesten Sinne wie Zuschüsse zur Renten- oder Krankenversicherung, nicht anders als für die Finanzierung von Kinderbetreuung, Schulen und Hochschulen, für den Ausbau des Straßen- und Schienennetzes sowie für Maßnahmen zu Gunsten der Erhöhung der inneren Sicherheit oder zumindest des Sicherheitsgefühls. Für die Einleitung eines verfassungsgerichtlichen Kontrollverfahrens finden sich nur selten Antragsteller. ${ }^{53}$ Typischerweise kommt als Antragsteller die Opposition im Bundestag in Betracht. Sie kann aber wegen der langen Verfahrensdauer eines Verfassungsgerichtsprozesses kaum mit einem schnellen für sie politisch verwertbaren Erfolg rechnen. Vielmehr droht umgekehrt eine verfassungsgerichtliche Entscheidung zu einem späteren Zeitpunkt ihre eigene Handlungsfähigkeit zu beeinträchtigen, wenn es ihr gelungen ist, die Regierungsmehrheit abzulösen.54 Das Bundesverfassungsgericht wird in den Verfahren über die Zulässigkeit einer Schuldenaufnahme des Bundes regelmäßig mit einer

51 Eine Übersicht zur Entwicklung der Nettokreditaufnahme im Bundeshaushalt bei Engels, D./Hugo, D.: Verschuldung des Bundes und rechtliche Schuldengrenzen, in: Die öffentliche Verwaltung, 60/11 (2007), 445-456, hier 446.

52 Siehe auch bereits Wieland, J.: Schuld(en) und Sühne? Wie kann ein anreizkompatibler und lösungsorientierter Umgang mit Altschulden aussehen?, in: Lange, J. (Hg.): Verflechtung verpflichtet: Die Reform der föderalen Finanzbeziehungen, Rehburg-Loccum, 2008, 145-150, hier 150.

53 In Betracht kommt eine abstrakte Normenkontrolle gem. Art. 93 Abs. 1 Nr. 2 GG, $\S \S 13$ Nr. 6, 76 ff. BVerfGG, vgl. BVerfGE 79, $311 \mathrm{ff}$, hier 326f.; Schlaich, K./Korioth, S.: Das Bundesverfassungsgericht, 7. Aufl., München, 2007, Rn. $126 \mathrm{f}$.

54 Wieland, J.: Staatsverschuldung als Herausforderung für die Finanzverfassung, in: Juristenzeitung, 61/15-16 (2006), 751-756, hier 753 f.; siehe auch Engels, D./Hugo, D., a. a. O., 453. 
großen Zahl einander widersprechender finanzwissenschaftlicher Gutachten konfrontiert, die eine Entscheidungsfindung nicht erleichtern. Ist das Gericht zu einer Entscheidung gelangt, liegt die Schuldenaufnahme häufig so lange zurück, dass sie in der Öffentlichkeit kaum noch auf Resonanz trifft. Es ist zu bezweifeln, ob sich diese Rahmenbedingen ändern würden, wenn die verfassungsrechtliche Schuldenregel anders formuliert würde. Da eine strikte Schuldenregel sich wegen der schwer vorhersehbaren tatsächlichen Entwicklungen im politischen Willensbildungsprozess kaum durchsetzen lassen wird, ${ }^{55}$ wird vermutlich jede neue Regel, wie die des Bundesfinanzministers, Raum für Ausnahmen und Reaktionen auf die Konjunkturentwicklung lassen müssen. Damit ist dann aber schon die Grundlage für Streitigkeiten darüber geschaffen, ob die Voraussetzungen für eine Ausnahme von der erlaubten strukturellen Regelverschuldung vorliegen.

Letztlich ist die Steuerungswirkung der Finanzverfassung in Fragen der Begrenzung der Kreditaufnahme des Staates überschaubar. Die richtige Höhe der Staatsverschuldung ist eher ein Problem von governance als eine Aufgabe, die sich mit den Mitteln rechtswissenschaftlich geleiteter Subsumption unter einer Verfassungsregel lösen ließe. Das spricht nicht notwendig gegen die Aufnahme einer neuen verfassungsrechtlichen Verschuldungsregel in das Grundgesetz. Es bleibt aber zu beachten, dass unter Geltung einer entsprechenden Vorschrift für die politischen Entscheidungsträger ein gewisser Anreiz besteht, sich damit zu begnügen, die Verfassungsvorgaben einzuhalten, statt sich der Frage zu stellen, ob nicht auch eine geringere Staatsverschuldung als von der Verfassung erlaubt möglich und wünschenswert wäre.

Für die Länder wirft jede verfassungsrechtliche Verschuldungsregel deshalb besondere Schwierigkeiten auf, weil sie nur in geringem Umfang über Einnahmen- und Ausgabenautonomie verfügen. ${ }^{56}$ Die Einnahmen der Länder bestimmt im Wesentlichen der Bund durch seine Steuergesetzgebung. Ihre Ausgaben sind zu einem erheblichen Teil durch Bundesgesetze zur Verwirklichung des sozialen Rechtsstaates determiniert. Wenn aber Steuereinnahmen und -ausgaben der Länder bundesrechtlich in großem Umfang vorgegeben sind, bleibt ihnen zum Ausgleich ihres Haushalts nicht selten nur der Ausweg in die Kreditaufnahme. Das

55 Vgl. Härtel, I., a. a. O., $441 \mathrm{f}$.

56 Vgl. Waldhoff, C.: Finanzautonomie und Finanzverflechtung in gestuften Rechtsordnungen, in: Veröffentlichungen der Vereinigung der Deutschen Staatsrechtslehrer, 66 (2007), 216-276, hier $237 \mathrm{ff}$., 260; Hey, J.: Finanzautonomie und Finanzverflechtung in gestuften Rechtsordnungen, in: Veröffentlichungen der Vereinigung der Deutschen Staatsrechtslehrer, 66 (2007), 277-334, hier 315; Bull, H.P.: Finanzausgleich im „Wettbewerbsstaat“, in: Die öffentliche Verwaltung, 52/7 (1999), 269-281, 275 f. 
ist eine wesentliche Ursache für die heute festzustellende hohe Verschuldung vieler Länder, die zum Teil bereits Haushaltsnotlagen hervorgerufen hat.

\section{Haushaltsnotlagen}

Mehrere Länder haben im Laufe der vergangenen Jahre so viele Schulden angehäuft, dass sie sich in einer Haushaltsnotlage befinden. Das Bundesverfassungsgericht hat 1992 im Saarland und in Bremen eine extreme Haushaltsnotlage festgestellt, aus der sich die beiden Länder nicht mehr aus eigener Kraft befreien könnten. Es hat deshalb aus dem Prinzip der Bundestreue die Pflicht des Bundes abgeleitet, den Haushaltsnotlagenländern aus ihrer Haushaltssituation herauszuhelfen. ${ }^{57} 2006$ hat das Gericht diese Rechtsprechung korrigiert. Es hat einen Antrag Berlins auf die Gewährung von Sanierungshilfe abgewiesen und betont, dass solche Hilfe nur nach Maßgabe eines strengen ultima-ratio-Prinzips in Betracht kommen könne. Sie sei nur dann verfassungsrechtlich zulässig und geboten, wenn die Haushaltsnotlage sowohl relativ - im Verhältnis zu anderen Ländern - als auch absolut - nach dem verfassungsrechtlich zugewiesenen Aufgabenbestand - extrem sei. Voraussetzung für Sanierungshilfe sei folglich ein bundesstaatlicher Notstand, der erst dann eingetreten sei, wenn das Land als verfassungsgerecht handlungsfähiger Träger staatlicher Aufgaben ohne fremde Hilfe in seiner Existenz bedroht sei. Das Land müsse alle ihm zur Verfügung stehenden Möglichkeiten der Abhilfe erschöpft haben, bevor eine Hilfe des Bundes als einziger Ausweg in Betracht komme. Insoweit obliege ihm die Darlegungs- und Begründungslast. ${ }^{58}$

Damit sind die Voraussetzungen für einen Anspruch auf Sanierungshilfe so hoch angesetzt, dass sie in der Staatspraxis kaum je erfüllt werden können. Das liegt wesentlich daran, dass das Bundesverfassungsgericht an den Kapitalmarkt mit seiner Entscheidung das Signal gesandt hat, dass im äußersten Notfall der Bund verhindern wird, dass ein Land seine Schulden nicht mehr zurückzahlen kann. Solange aber der Bund auf diese Weise die Schulden der Länder absichert, sind diese weiter kreditfähig und werden nicht in die Existenz bedrohende Lage geraten, die Voraussetzung für eine Sanierungshilfe nach der neueren Rechtspre-

58 BVerfGE 116, 327 ff., hier 377; hierzu auch Wieland, J.: Die ,extreme Haushaltsnotlage“ in der Rechtsprechung des Bundesverfassungsgerichts und der Normenkontrollantrag Berlins, in: Baßeler, U./Heintzen, M./Kruschwitz, L. (Hg.): Berlin - Finanzierung einer Metropole, Berlin, 2006, 173-184, hier 173. 
chung ist. ${ }^{59}$ Im Ergebnis können die Länder also nach der Verfassungsrechtsprechung weiter Kredite aufnehmen, werden jedoch vom Bund keine Sanierungshilfe erhalten. Das erklärt ihren Widerstand gegen strikte Schuldenregelung, die ihre Handlungsfähigkeit deutlich beschränken würde. Aus dem gleichen Grunde dürfte auch der von Finanzwissenschaftlern gemachte Vorschlag, Insolvenzregelungen für Privatpersonen in modifizierter Form auf Gebietskörperschaften zu übertragen, ${ }^{60}$ in der Staatspraxis keinen Erfolg haben. Auch modifizierte Insolvenzregelungen würden nämlich die Möglichkeit der Länder zur weitere Kreditaufnahme für die Zukunft erheblich einschränken, wenn nicht sogar beseitigen. Gerade die so erzwungene Haushaltsdisziplin ist jedoch für Länder in Haushaltsnotlagen solange nicht attraktiv, wie ihnen der Bund nicht Hilfe beim Abbau ihrer übermäßigen Verschuldung geleistet hat. Eine Schuldenhilfe des Bundes an Länder in Haushaltsnotlagen wird also der Preis für eine neue verfassungsrechtliche Schuldenregel sein.

Eine Schuldenhilfe wird allerdings nur dann geleistet werden, wenn die Notlagenländer selbst alle zumutbaren Sparanstrengungen unternehmen. Davon wird nur dann die Rede sein können, wenn sie ihre Vermögensreserven aktivieren und ihr Ausgabenniveau unter den Durchschnitt vergleichbarer Länder absenken. Länder, die von der bundesstaatlichen Gemeinschaft Hilfe bei der Tilgung ihrer übermäßigen Schulden erhalten, werden sich auch für die Zeit der Haushaltssanierung mit erheblichen Einschränkungen ihrer Haushaltsautonomie einverstanden erklären müssen. ${ }^{61}$ Ziel der Sanierungshilfe sollte es sein, den Verschuldungsgrad der Haushaltsnotlagenländer so weit abzusenken, dass sie bei strikter Sparsamkeit eine realistische Chance haben, die verbleibenden Schulden aus eigener Anstrengung heraus abzutragen.

Letztlich setzt eine dauerhafte Sanierung der Haushalte von Bund und Ländern aber eine Anpassung der primären Steuerverteilung an die Verhältnisse im wiedervereinigten Deutschland voraus. Gegenwärtig ist der Maßstab des Art. 107 Abs. 1 GG für die primäre Steuerverteilung vorrangig das örtliche Aufkommen einer Steuer. Dieser Maßstab war 1968/69, als die Finanzverfassung reformiert wurde, deshalb vertretbar, weil die Finanz- und Wirtschaftskraft der westdeut-

59 Siehe bereits Wieland, J.: Schuld(en) und Sühne?, a. a. O., $147 \mathrm{f}$.

60 Vgl. Blankart, C.B./Fasten, E.R./Klaiber, A.: Föderalismus ohne Insolvenz?, in: Humboldt Forum Recht 2008, Beitrag 3, abrufbar unter http://www.humboldt-forum-recht.de; zur kommunalen Insolvenz Firelinghaus, S.N.: Die kommunale Insolvenz als Sanierungsansatz für die öffentlichen Finanzen, in: Die öffentliche Verwaltung, 60/15 (2007), 636-647.

61 Vgl. Wieland, J.: Staatsverschuldung als Herausforderung, a. a. O., 756. 
schen Länder im Großen und Ganzen vergleichbar war. ${ }^{62}$ Unter diesen Umständen erschien auch den finanzschwächeren Ländern eine Finanzordnung sinnvoll, die mit der primären Steuerverteilung an das örtliche Aufkommen einer Steuer anknüpfte und verbleibende Defizite gegenüber einer aufgabengerechten Finanzverteilung durch den horizontalen Länderfinanzausgleich und durch Bundesergänzungszuweisungen, die als Spitzenausgleich konzipiert sind, bewältigte. ${ }^{63}$ Insbesondere nach der Wiedervereinigung Deutschlands sind die tatsächlichen Voraussetzungen für ein System der Steuerverteilung nach dem primären Aufkommen aber nicht mehr gegeben. Die Unterschiede in der Wirtschafts- und Finanzkraft der Länder sind beachtlich. Das kommt besonders deutlich, aber keineswegs ausschließlich darin zum Ausdruck, dass die Steuerkraft der ostdeutschen Länder nur $40 \%$ des Bundesdurchschnitts beträgt. ${ }^{64}$ Mit einem solchen örtlichen Aufkommen lässt sich aber auf Dauer kein durch Bundesgesetze einheitlich auf $100 \%$ festgelegtes Ausgabenniveau für sozialstaatliche Leistungen finanzieren. Diese Spannungslage wird gegenwärtig noch durch Bundesergänzungszuweisungen und den Solidarpakt II überdeckt. Wegen der degressiven Ausgestaltung der Sonderbundesergänzungszuweisungen für ostdeutsche Länder wird jedoch in den nächsten Jahren immer deutlicher werden, dass das System der Steuerverteilung in Deutschland grundlegend in Richtung auf eine aufgabengerechte Finanzausstattung reformiert werden muss. Dafür ist in das Gedächtnis zu rufen, dass Einkommensteuer, Körperschaftssteuer und Umsatzsteuer als Gemeinschaftsteuern nationale Steuern sind. Sie knüpfen an die Wertschöpfung in ganz Deutschland an. Dementsprechend steht ihr Ertrag dem Bund und allen Ländern gemeinsam zu. ${ }^{65}$ Das örtliche Aufkommen an Gemeinschaftssteuern allein ist angesichts der Unterschiede zwischen der Wirtschafts- und Finanzkraft der verschiedenen Teile Deutschlands kein sachgerechter Maßstab für die primäre Steuerverteilung mehr. Das gilt nicht zuletzt deshalb, weil durch steuerliche Gestaltungen gerade im Bereich der Unternehmenssteuern das örtliche Aufkommen verlagert werden kann, ohne dass sich etwas am Ort der Wertschöpfung

62 Zur Geschichte des Art. 107 GG Heun, W., in: Dreier, H. (Hg.): Grundgesetz: Kommentar, Bd. 3, Tübingen, 2000, Art. 107, Rn. 4,; Huber, P. M., in: Mangoldt, H. v./Klein, F./Starck, C. (Hg.): Kommentar zum Grundgesetz, Bd. 3, 5. Aufl., München, 2005, Art. 107, Rn. 3 f.

63 Wieland, J.: Finanzverfassung, Steuerstaat und föderaler Ausgleich, in: Badura, P./Dreier, H. (Hg.): Festschrift 50 Jahre Bundesverfassungsgericht, Bd. 2, Tübingen, 2001, 771-801, hier $786 \mathrm{ff}$.

64 Hierzu Höfling, W.: Haushalts- und Finanzverfassung in der Krise - Steuerungsschwächen, Fehlanreize, Reformoptionen, in: Der Staat 46/2, (2007), 163-181, 179.

65 Vgl. Heun, W., in: Dreier, H. (Hg.): Grundgesetz: Kommentar, Bd. 3, Tübingen, 2000, Art. 106, Rn. $17 \mathrm{ff}$. 
änderte. Eine aufgabengerechte Verteilung des Aufkommens der Gemeinschaftssteuern wird sich deshalb in Zukunft nur erreichen lassen, wenn die Bedeutung des örtlichen Aufkommens relativiert wird.

Bei realistischer Betrachtung kann eine Reform der primären Steuerverteilung allerdings politisch nur längerfristig umgesetzt werden. Dagegen sind die Probleme der Staatsverschuldung bereits kurzfristig so drängend, dass ihre Lösung keinen Aufschub duldet. Der Hessische Ministerpräsident Roland Koch hat deshalb einen Generationenvertrag über den Weg aus der Schuldenfalle vorgeschlagen. ${ }^{66}$ Danach sollen Bund und Länder sowie gegebenenfalls auch die Kommunen auf der Basis eines Staatsvertrags ihre gesamten Kreditmarktschulden in einen gemeinsamen Fonds einbringen. Dessen Laufzeit soll 50 Jahre umfassen. Der Fonds soll gegenüber den Kreditgebern gesamtschuldnerisch haften. Im Innenverhältnis sollen Bund und Länder gegenüber dem Fonds entsprechend den von ihnen eingebrachten Schuldenanteilen haften. Dieses Konzept ist mit der Erwartung verbunden, dass gemeinsam betriebene Kreditaufnahmen in der Regel $\mathrm{zu}$ geringeren Finanzierungskosten führten. Auch sollen die längere Laufzeit der Kredite sowie ein hervorragendes Schuldenmanagement zusätzliche liquide Mittel freisetzen. Auf diesem Wege soll eine Effizienzrendite erzielt werden, aus der Sanierungshilfen für Länder finanziert werden sollen, die in absehbarer Zeit keinen dauerhaft ausgeglichenen Haushalt erreichen können. Bund und Länder sollen sich zu einer Beteiligung an der Gesamtannuität des Fonds entsprechend dem Anteil der von ihnen eingebrachten Schulden verpflichten. Koch räumt allerdings ein, dass die so berechneten Annuitäten die Finanzierungsmöglichkeiten der Länder voraussichtlich übersteigen werden. ${ }^{67}$ Bisher haben die Länder nämlich ihre Tilgungsraten regelmäßig durch die Aufnahme neuer Kredite finanziert. Die Nutzung aufwachsender Einnahmen und weitere Konsolidierungsmaßnahmen zur Finanzierung der vorgesehenen Tilgung der bestehenden Schulden hält auch Ministerpräsident Koch für einen Kraftakt, der bei einem sofortigen Verbot der Aufnahme neuer Kredite für den allgemeinen Haushalt kaum gelingen könne. Bund und Länder sollen deshalb das Recht erhalten, in einer Übergangszeit von höchstens 10 Jahren sinkende Anteile der Tilgungsraten durch die Aufnahme neuer Kredite zwischenzufinanzieren. Der Bund oder ein Land müsste im ersten Jahr neben seinen Zinsverpflichtungen gegenüber dem Fonds mindes-

66 Koch, R.: Hessisches Modell zur Lösung des Verschuldungsproblems. Aufgabenfinanzierung auf Kosten zukünftiger Generationen beenden - Generationenvertrag für einen Weg aus der Schuldenfalle -, K-Drs. 107 vom 23.04. 2008.

67 Ebd., 4. 
tens $10 \%$ der Tilgungssumme des auf 50 Jahre gerechneten Kredites ohne neue Kreditaufnahme finanzieren. Nach 10 Jahren müssten $100 \%$ der Tilgungssumme ohne die Aufnahme neuer Kredite finanziert werden. Sondertilgungen sollen möglich sein. Für den Fall, dass einzelne Länder ungeachtet aller Eigenanstrengungen und Sparbemühungen keinen nachhaltig ausgeglichenen Haushalt vorlegen könnten, sollen sie Zuschüsse zu den eigenen Zinszahlungen erhalten. Diese Zinshilfen würden zwar längerfristig gewährt, ihr Ende müsste allerdings festgeschrieben und die Hilfe müsste degressiv ausgestaltet sein. Nach Kochs Plan könnte der Schuldenplan seine Aufgabe nur dann erfüllen, wenn die Haushalte von Bund und Ländern dauerhaft schuldenfrei blieben. Deshalb dürften weder der Bund noch die Länder Kredite aufnehmen, die nicht im Staatsvertrag vereinbart worden seien. Nur in eng begrenzten und eindeutig definierten Ausnahmefällen (Naturkatastrophen, Einbrüche bei den Steuereinnahmen) soll dem Plan nach auch weiterhin eine staatliche Kreditaufnahme vorübergehend möglich sein. Der für das Funktionieren des Verschuldungsfonds erforderliche Bundeszuschuss müsste sich nach der Schätzung von Ministerpräsident Koch auf 3 bis 4 Mrd. € pro Jahr belaufen. Hinzu kämen Zinszuschüsse für strukturschwache Länder in Höhe von „1 Milliarde plus x Euro“ pro Jahr. ${ }^{68}$

Gerade die letztgenannten Zahlen machen deutlich, warum der Plan eines Schuldenfonds zwar für ein wirtschafts- und finanzstarkes Land wie Hessen attraktiv erscheint, nicht jedoch für den Bund, der alleine im Vertrauen auf Effizienzgewinne die Verpflichtung zur Zahlung von mehr als $5 \mathrm{Mrd}$ € pro Jahr an Zuschüssen übernehmen müsste. Richtig dürfte sein, dass ein Verzicht auf die Aufnahme neuer Kredite nicht von heute auf morgen, sondern nur nach Ablauf einer längeren Übergangszeit erreichbar erscheint. Auch Koch kommt jedoch nicht ohne Ausnahmen von Verschuldungsverbot aus. Ob diese Ausnahmen vorliegen, würde zwischen den Beteiligten mit an Sicherheit grenzender Wahrscheinlichkeit streitig sein. Das gilt insbesondere für ein so ,weiches“ Merkmal wie einen Einbruch bei den Steuereinnahmen. Aus Sicht der finanzstarken Länder hat das Modell allerdings den wichtigen Vorteil, dass die finanziellen Lasten der Fondslösung von den verschuldeten Ländern und vom Bund, nicht jedoch von den finanzstarken Ländern zu tragen wären. ${ }^{69}$ Das lässt die praktischen Chancen auf eine Realisierung des Modells als gering erscheinen. Bemerkenswert ist aber,

68 Ebd., 3.

$69 \mathrm{Zu}$ den divergierenden Interessen von Bund sowie Geber- und Nehmerländern etwa Härtel, I., a. a. O., $442 \mathrm{f}$. 
dass der Ministerpräsident eines finanzstarken Landes mit dem Plan für einen Entschuldungsfonds die Notwendigkeit anerkennt, die Altschuldenproblematik in einer gemeinsamen Anstrengung des Bundesstaates zu lösen. Sollte ein vergleichbares Modell Erfolg haben, wäre allerdings zwingend eine andere Lastenverteilung erforderlich, an der sich auch die finanzstarken Länder beteiligen müssten. Längerfristig wird zudem kein Weg an einer Neuordnung der primären Steuerverteilung vorbeiführen, weil anderenfalls zumindest einige Länder nach kurzer Zeit entweder faktisch handlungsunfähig würden oder neue Schulden aufnehmen müssten. Es ist noch einmal zu betonen, dass sich aus einem Steueraufkommen von $40 \%$ des Bundesdurchschnitts Ausgaben sozialstaatlicher Natur in Höhe von 100 \% des Bundesdurchschnitts nicht finanzieren lassen. ${ }^{70}$

\section{Bundessteuerverwaltung}

Im Rahmen der Kommissionsverhandlungen hat der Bund lange Zeit nachdrücklich die Einführung einer Bundessteuerverwaltung gefordert. Er hat auf Gutachten hingewiesen, nach denen durch diesen Schritt erhebliche Effizienzgewinne erzielt werden könnten. ${ }^{71}$ Auch verhindere eine Bundessteuerverwaltung einen verdeckten Steuerwettbewerb zwischen den Ländern, in dem mit dem Verzicht auf Außenprüfungen und ähnlichen Maßnahmen versucht werden könnte, Unternehmen zur Ansiedlung in einem Land zu bewegen. ${ }^{72}$ Der Bund verweist in diesem Zusammenhang darauf, dass schon der Parlamentarische Rat 1949 eine Bundessteuerverwaltung einführen wollte. Dazu ist es nur deshalb nicht gekommen, weil die westlichen Alliierten eine zu große Machtballung beim Bund befürchteten und deshalb dafür Sorge trugen, dass ein erheblicher Teil der Steuerverwaltung bei den Ländern angesiedelt wurde. ${ }^{73}$ Dieses Vorgehen der Alliierten hat schon vor bald 60 Jahren deutlich gemacht, dass die Frage einer Bundessteuerverwaltung eine Machtfrage par excellence ist, wie es Peter Michael Huber bei der zweiten Anhörung der Kommission zur Modernisierung der Bund-Länder-

70 Vgl. bereits Wieland, J.: Staatsverschuldung als Herausforderung, a. a. O., 755.

71 Bundesrechnungshof: Position des Bundesrechnungshofes zur Bundessteuerverwaltung, K-Drs. 110 vom 28.04. 2008; siehe auch Bundesbeauftragter für Wirtschaftlichkeit in der Verwaltung: Probleme beim Vollzug der Steuergesetze, Stuttgart, 2006.

$72 \mathrm{Zu}$ diesem „Steuerwettbewerb“ etwa bereits Der Spiegel vom 14.06. 2004, 24 ff.; siehe auch Capital vom 13.05. 2004, 86, sowie vom 27.05. 2004, 114.

73 Vgl. dazu Oeter, S.: Die Finanzverwaltung, a.a. O., 1 ff. 
Finanzbeziehungen am 8. November 2007 in Berlin betont hat. ${ }^{74}$ Deshalb überrascht es nicht, dass fast alle Länder sich dem Wunsch des Bundes nach der Einführung einer Bundessteuerverwaltung nachdrücklich widersetzt haben. Dieser Widerstand hat den Bundesfinanzminister dazu bewogen, sich auf die Forderung nach besseren Möglichkeiten zur technischen Koordinierung der Länder zu beschränken. ${ }^{75}$ Diese Forderung ist so abstrakt formuliert, dass sie viel Raum zur Interpretation lässt. Die Länder werden einer gleichberechtigten Koordinierung sicherlich eher zustimmen können als einer vom Bund gesteuerten Koordinierung. Immerhin könnte in der Konzentration auf die Einführung neuer Möglichkeiten zur technischen Koordinierung eine Lösung des Grundsatzstreites um die Einführung einer Bundessteuerverwaltung liegen, die als solche angesichts der weitgehenden Ablehnung des Vorschlags des Bundes im Kreise der Länder keine Chance auf Realisierung hätte.

\section{Ausblick}

Bereits der vorstehende Überblick hat gezeigt, dass eine Einigung von Bund und Ländern auch in den wenigen hier aufgezeigten Bereichen Begrenzung der Staatsverschuldung, Lösung der Altschuldenproblematik und Einführung einer Bundessteuerverwaltung äußerst schwierig erscheint. Ein Erfolg der Kommissionsarbeit ist deshalb keineswegs sicher. In jedem Fall werden die Chancen für eine Einigung erst nach der Landtagswahl in Bayern am 28. September 2008 deutlich ansteigen. Am wahrscheinlichsten dürfte gegenwärtig sein, dass bei einem positiven Abschluss der Kommissionsberatungen das Ergebnis eine relativ milde Regel zur Begrenzung der Neuverschuldung im Austausch gegen bundesstaatliche Hilfe bei der Bewältigung der Altschuldenproblematik sein könnte. Auch eine effizientere Zusammenarbeit in der Steuerverwaltung zwischen Bund und Ländern käme als weiteres Resultat der Föderalismusreform II in Betracht. Wesentlich mehr zu erwarten, dürfte angesichts der politischen Gegebenheiten eher unrealistisch sein. In jedem Fall wird der Bundesstaat in Deutschland auch nach einem erfolgreichen Abschluss der zweiten Stufe der Bundesstaatsreform unvollkommen bleiben.

74 Huber, P.M., in: Kommission von Bundestag und Bundesrat zur Modernisierung der Bund-LänderFinanzbeziehungen: Komm.-Prot. 08 vom 08. 11. 2007, 216.

75 Bundesministerium der Finanzen: Positionspapier zur Notwendigkeit weiterer gesetzlicher Befugnisse des Bundes zur Steigerung der Effizienz der Steuerverwaltung, K-Drs. 109 vom 28.04. 2008. 